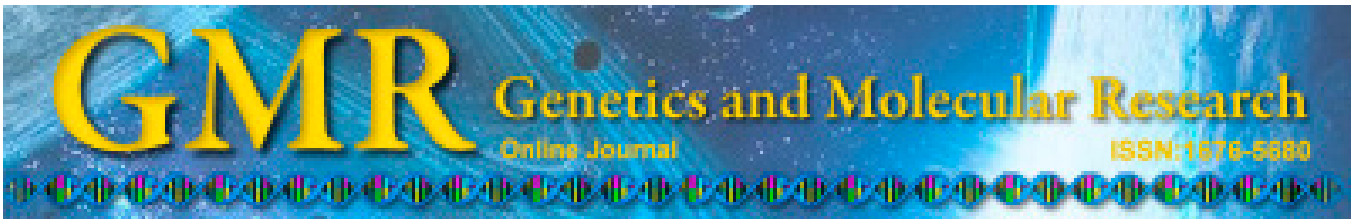

\title{
Characterization and expression analysis of Toll-like receptor 3 cDNA from Atlantic salmon (Salmo salar)
}

\author{
R. Vidal ${ }^{1}$, R. González ${ }^{1}$ and F. Gil ${ }^{2}$ \\ ${ }^{1}$ Laboratorio de Ecologìa Molecular, Genòmica y Estudios Evolutivos, \\ Departamento de Biologìa, Facultad of Quìmica y Biologìa, \\ Universidad de Santiago, Santiago, Chile \\ ${ }^{2}$ Laboratorio de Microbiologìa Molecular, Facultad de Ciencias Biològicas, \\ Universidad Andrés Bello, Santiago, Chile \\ Corresponding author: R. Vidal \\ E-mail: ruben.vidal@usach.cl
}

Genet. Mol. Res. 14 (2): 6073-6083 (2015)

Received October 20, 2014

Accepted January 19, 2015

Published June 8, 2015

DOI http://dx.doi.org/10.4238/2015.June.8.5

\begin{abstract}
Innate pathway activation is fundamental for early antiviral defense in fish, but currently there is insufficient understanding of how salmonid fish identify viral molecules and activate these pathways. The Toll-like receptor (TLR) is believed to play a crucial role in host defense of pathogenic microbes in the innate immune system. In the present study, the full-length cDNA of Salmo salar TLR3 (ssTLR3) was cloned. The ssTLR3 cDNA sequence was $6071 \mathrm{bp}$ long, containing an open reading frame of $2754 \mathrm{bp}$ and encoding 971 amino acids. The TLR group motifs, such as leucine-rich repeat (LRR) domains and Toll-interleukin-1 receptor (TIR) domains, were maintained in ssTLR3, with sixteen LRR domains and one TIR domain. In contrast to descriptions of the TLR3 in rainbow trout and the murine (TATA-less), we found a putative TATA box in the proximal promoter region $29 \mathrm{bp}$ upstream of the transcription start point of ssTLR3. Multiple-sequence alignment analysis of the ssTLR3 protein-coding sequence with other
\end{abstract}


known TLR3 sequences showed the sequence to be conserved among all species analyzed, implying that the function of the TLR3 had been sustained throughout evolution. The ssTLR 3 mRNA expression patterns were measured using real-time PCR. The results revealed that TLR3 is widely expressed in various healthy tissues. Individuals challenged with infectious pancreatic necrosis virus and immunostimulated with polyinosinic:polycytidylic acid exhibited increased expression of TLR3 at the mRNA level, indicating that ssTLR3 may be involved in pathogen recognition in the early innate immune system.

Key words: Toll-like receptor; TLR3; Innate immunity; Atlantic salmon; Infections pancreatic necrosis virus

\section{INTRODUCTION}

Vertebrates have evolutionarily evolved an innate and adaptive immune system to eliminate invading pathogens. The innate immune system is crucial as the first line of protection against invasion by pathogens and contributes the pivotal signals for the activation of adaptive immune responses, foremost to immunological memory (Magnadottir, 2006). Innate immune activation is provoked upon pathogen recognition by a group of pattern recognition receptors that identify the unique pathogen-associated molecular patterns (PAMPs) of microorganisms, which are indispensable for their survival (Medzhitov, 2007).

Among the known pattern recognition receptors for microbial substances, Toll-like receptors (TLRs) comprise a family of single-pass transmembrane receptors $(>12)$ that can identify various classes of PAMPs, such as peptidoglycans, lipoproteins, double-stranded viral RNA, LPS, and unmethylated bacterial CpG DNA (Akira and Takeda, 2004). In general, TLRs are represented by an extracellular N-terminus with a leucine-rich repeat region (LRR) and a conserved cytoplasmic C-terminus with a Toll-interleukin-1 receptor (TIR) domain (Matsushima et al., 2007). The TIR-conserved amino acid regions have been shown to be implicated in the signaling and localization of TLRs (Sarkar et al., 2003; Funami et al., 2004), while the LRR has been implicated in pathogen detection (Bell et al., 2003). TLR family components are expressed by cells of the immune system and specialized cells, such as neutrophils, macrophages, dendritic cells, vascular endothelial cells, and intestinal epithelial cells (Skjaeveland et al., 2008). Moreover, TLRs trigger the innate immune system, mobilizing several transcription factors (NF- $\mathrm{KB}, \mathrm{AP} 1$, and IRFs), stimulating in turn the expression of pro-inflammatory cytokines and type I interferon (IFN) (Kawai and Akira, 2007). In teleosts, 17 different TLRs have been described and specific teleost TLRs that share functional characteristics with their mammalian counterparts have been revealed (Palti, 2011). Nonetheless, singular features of teleostean TLR cascades have also been reported (Rebl et al., 2007).

Atlantic salmon (Salmo salar) farmers worldwide are faced with viral infections, which can destroy a significant part of production each year and result in substantial economic losses. To efficiently manage disease problems in Atlantic salmon aquaculture, information on how fish respond to pathogen exposure is needed. In the present study, we describe the genomic organization, expression pattern, and regulation of the $S$. salar TLR3 (ssTLR3) ortholog in Atlantic salmon, one of the most important aquaculture fish species in the world. In fish, TLR3 has been described in zebrafish, catfish, fugu, and rainbow trout (Palti, 2011), 
but description and regulation of gene expression to viral challenge have not been previously characterized in Atlantic salmon.

A cDNA library from Atlantic salmon resulted in the isolation of cDNA encoding ssTLR3 (Cepeda et al., 2011). This cDNA was cloned, sequenced, and aligned with other TLR3 sequences. Using sequence alignment, a gene tree was reconstructed. In the final step, we studied the tissue distribution and mRNA expression pattern of ssTLR3 during viral infection.

\section{MATERIAL AND METHODS}

\section{Cloning and sequencing of ssTLR3 cDNA}

Full-length ssTLR3 cDNA was characterized using an expressed sequence tag analysis of a library constructed from Atlantic salmon head kidney (Cepeda et al., 2011).

\section{Peptide sequence alignment and comparative genomic analyses}

The open reading frames (ORFs) and their protein translation were predicted using the ExPASy Translate tool (http://us.expasy.org/). The mass of the hypothetical proteins was obtained using the Prot-Param tool (http://www.expasy.org/protparam/) and the signal peptide was detected using SignalP (http://www.cbs.dtu.dk/services/SignalP/). Homologous genes from other species were identified for ssTLR3 using BLASTx (NCBI; Altschul et al., 1990) global alignment (accession numbers of the sequences are given in Table 1). These alignments were used to generate a consensus gene tree using the molecular evolution genetics analysis (MEGA) v5.0 software (Tamura et al., 2011). The gene tree was reconstructed using the neighbor-joining algorithm (Poisson's correction and exclusion of gaps), with 10,000 repetitions for bootstrap analysis.

\begin{tabular}{|c|c|c|c|}
\hline & \multicolumn{3}{|c|}{ Full ssTLR3 sequence } \\
\hline & Identity (\%) & Similarity (\%) & Amino acid accession No. \\
\hline omTLR3 ${ }^{a}$ & 94.98 & 96.62 & AAX68425.1 \\
\hline trTLR3 & 60.07 & 74.27 & AAW69373.1 \\
\hline ipTLR3 & 58.85 & 74.52 & AEI59664.1 \\
\hline drTLR3 & 58.05 & 74.05 & NP_001013287.2 \\
\hline btTLR3 & 50.06 & 66.59 & ABN̄71659.1 \\
\hline ssTLR3 & 48.93 & 67.42 & NP_001090913.1 \\
\hline ggTLR3 & 49.04 & 67.23 & NP_001011691.3 \\
\hline mmTLR3 & 48.70 & 67.12 & AĀ̄ 99937.1 \\
\hline hsTLR3 & 48.07 & 65.97 & NP_003256.1 \\
\hline
\end{tabular}

${ }^{a}$ Trout (om), fugu (tr), catfish (ip), zebrafish (dr), bovine (bt), Salmo salar (ss), chicken (gg), mouse (mm), and human (hs). All accession numbers are from GenBank.

\section{Domain organization}

The simple modular architecture research tool (SMART) (Schultz et al., 1998; Letunic et al., 2009), domain search algorithm, SignalP 4.0 (Petersen et al., 2011), and MINNOU 
(Cao et al., 2006; http://minnou.cchmc.org/) programs were used to characterize peptide architecture based on amino acid sequences and to search for protein family (Pfam) domains, signal sequences, and transmembrane domains. To evaluate possible transcription factor binding sites, we used the TFSEARCH (v1.3) program with scores higher than 85.0 (Heinemeyer et al., 1998).

\section{Tissue-specific expression in Atlantic salmon}

Total RNA was extracted from Atlantic salmon head kidney, spleen, heart, liver, intestine, and muscle using TRIzol reagent (Invitrogen, USA) and the RNeasy Lipid tissue mini kit (Qiagen, Germany), following the manufacturer protocol. The RNA pellet was washed with $80 \%$ ethanol, air-dried, and dissolved in RNase-free $\mathrm{H}_{2} \mathrm{O}$. The concentration/purity and integrity of RNA samples were evaluated using spectrophotometry (Nanodrop) and an Agilent Bioanalyzer 2100 , respectively. The RNA was stored at $-80^{\circ} \mathrm{C}$.

Expression of Atlantic salmon ssTLR3 in different tissues was evaluated using realtime PCR (RT-qPCR) with the primers ssTL3-F (5'-TTCTGGACTTTGTACCCGCC-3') and ssTLR3-R (5'-ACACAGGAGGCAGATGGTTG-3') and the Applied Biosystem 7300 system. For RT-qPCR, $1 \mu \mathrm{g}$ total RNA per sample was transcribed into cDNA using the Verso $^{\mathrm{TM}}$ cDNA kit (ABgene, Surrey, UK), and random hexamers (400 ng/ $\mu \mathrm{L}$ ). RT-qPCR mixtures (final volume $20 \mu \mathrm{L}$ ) containing either $5 \mu \mathrm{L}$ or $2 \mu \mathrm{L}$ (for the reference genes and HOX) diluted $(1 / 20)$ cDNA, $0.5 \mu \mathrm{M}$ of each primer, and $10 \mu \mathrm{L}$ Absolute ${ }^{\mathrm{TM}} \mathrm{QPCR} \mathrm{SYBR}^{\circledR}$ Green mix (ABgene) were incubated at $95^{\circ} \mathrm{C}$ for $15 \mathrm{~min}$, followed by 40 cycles of $15 \mathrm{~s}$ at $95^{\circ} \mathrm{C}, 15 \mathrm{~s}$ at the specific primer pair annealing temperature, and $15 \mathrm{~s}$ at $72^{\circ} \mathrm{C}$. Then, a melt curve of $0.5^{\circ} \mathrm{C}$ increments from $75^{\circ}$ to $90^{\circ} \mathrm{C}$ was performed to confirm amplification of a unique product in each reaction. $\mathrm{Ct}$ values were normalized with respect to the reference genes 18S rRNA and EF1- $\alpha$ (Cofre et al., 2014).

\section{Expression of ssTLR3 after viral challenge and polyinosinic:polycytidylic acid (poly I:C) stimulation}

Tissue samples from control and infectious pancreatic necrosis virus (IPNV)-infected fish originated from our previous study Cepeda et al. (2011). The head kidney was removed from infected and control fishes at 1,5, and 21 days post-infection. To examine expression of ssTLR3 following Poly I:C (Sigma, P9582) stimulation, 20 fish of approximately $40 \mathrm{~g}$ each were acclimated for 7 days and fed daily with an amount of commercial feed pellet corresponding to $1 \%$ of their body weight for $1 \mathrm{~h}$, after which the water was changed using the trap technique to avoid toxic levels of ammonium that could affect gene expression. The 20 fish were randomly divided into two groups, one group was intraperitoneally injected with $100 \mu \mathrm{L}$ phosphate-buffered saline and the other group was intraperitoneally injected with $100 \mu \mathrm{L} 2$ $\mu \mathrm{g} / \mu \mathrm{L}$ PolyI:C. After $24 \mathrm{~h}$, the fish were anaesthetized and head kidneys were sampled from both treated and control fishes.

Expression of ssTLR3 was evaluated using RT-qPCR with similar conditions and primers as described above. Gene expression results were analyzed using the Pfaffl method (Pfaffl, 2001). Ct values were normalized with respect to the reference genes 18S rRNA and EF1- $\alpha$ (Cofre et al., 2014). The Kruskal-Wallis test was used to determine whether gene expression differed significantly between time points $(\mathrm{P}<0.05$; R package V. 2.15.3). 


\section{RESULTS}

\section{ssTLR3 gene identification and sequence analysis}

The ssTLR3 cDNA sequence (GenBank accession No. KP231342) was 6071 bp in length, in addition to a 26-bp 5'-untranslated region (UTR), a 3'-UTR of $1802 \mathrm{bp}$, and an ORF of $2754 \mathrm{bp}$, encoding a 917-amino acid peptide. Seven instability motifs (ATTTA) were observed in the 3'-UTR of the salmon (Figure 1). The theoretical molecular weight of ssTLR3 was $102,605 \mathrm{kDa}$.

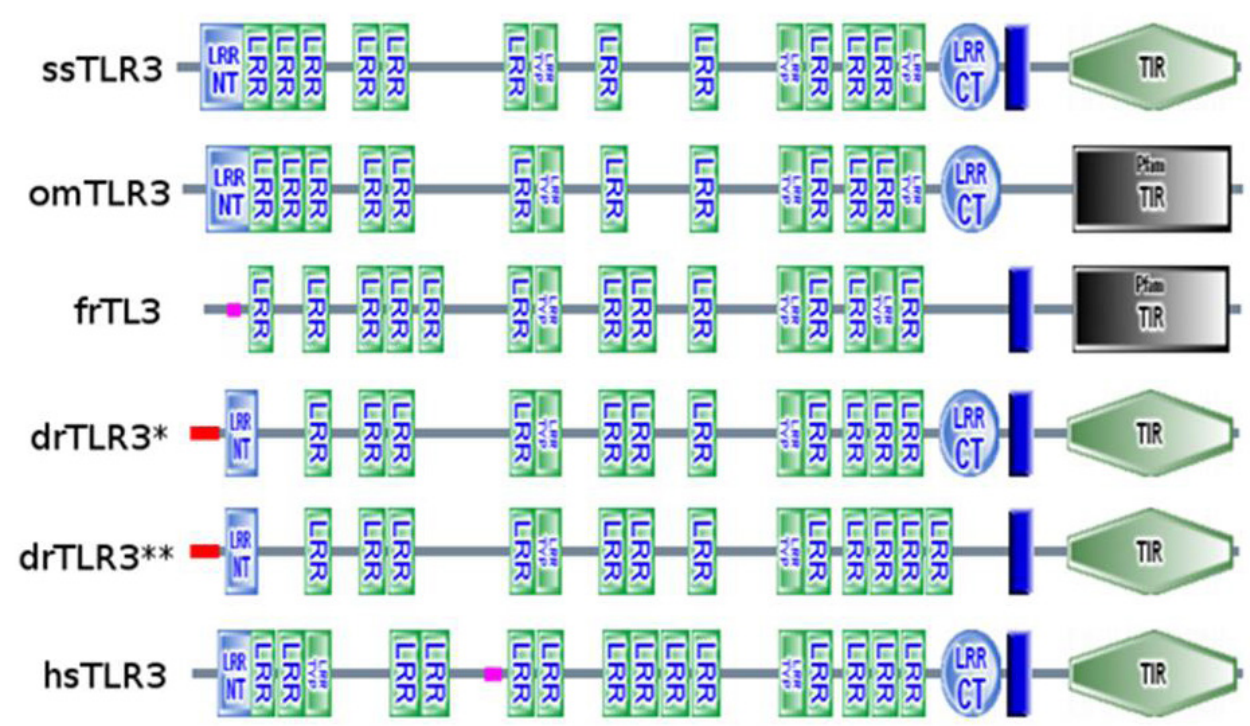

Figure 1. Schematic comparative domain organization of ssTLR3 with trout (om), fugu (fr), zebrafish (dr), and human (hs). The domains were predicted using SMART. LRR: leucine-rich repeat; LRR_TYP: leucine-rich repeat typical; TIR: Toll/IL-1 receptor; NT:N (nitrogen/amino)-terminal; CT:C (carboxyl)-terminal.

\section{Domain organization of ssTLR3}

This analysis located the extracellular domain containing the LRRs and the transmembrane and TIR domains. Computer analysis of the receptor structure in the ssTLR3 sequence, using the SMART program, predicted 16 LRRs, including the $\mathrm{C}$ - and $\mathrm{N}$-terminal regions, and a TIR domain (Figures 1 and 2). A signal peptide with a probability of at least $95 \%$ was also predicted in the N-terminus of the receptor using the SignalP 4.0 program. The ssTLR3 LRRs were distributed similarly to the LRRs identified in trout, fugu, zebrafish, and humans. Various hypothetical transcription factor-binding sites were determined in the ssTLR3 promoter region, using the TFSEARCH program (data not shown), such as an interferon-stimulated response element (ISRE) $608 \mathrm{bp}$ upstream of the transcription initiation start point. A hypothetical TATA box was recognized in the proximal promoter region 29 bp upstream of the transcription start point. 
SSTLR3 OMTLR3 f rTLR3 hSTLR3

SSTLR3 OMTLR3 f rTLR3 hSTLR3

SSTLR3 OmTLR3 f rTLR3 hSTLR3

SSTLR3 OMTLR3 f rTLR3 hSTLR3

SSTLR3 OITLR3 f rTLR3 hSTLR3

SSTLR3 OMTLR3 f TTLR3 hSTLR3

SSTLR3 OITLR3 f rTLR3 hSTLR3

SSTLR3 omTLR3 f rTLR3 hSTLR3

SSTLR3 OmTLR3 f rTLR3 hSTLR3
MNWPDI ILILLAVNLGDLITGPTLCHASOKHOKSECQVRNGWADCRHLRLKEIPPNLPWN MNWPDI ILILLAVNLGDLITGPTLCHASQKROKSECQVRNGWADCSHLRLKEIPPNLPWN .................... - RRHAVYGQKKTSCVVQGSSADCSHLSLSSIPPDLPRN ........ MRQTLPCIYFWGGLLPFGMLCASSTTKCTVSHEVADCSHLKLTQVPDDLPTN $\ldots{\text {.** } * * * * * * \ldots:^{*}: * * *}^{* *}$

ITGLDVSHNRLVELPPASLATYPGLVHLDVGFNSLTKLEDSLCQTLGLLRTLTVQHNEVH ITGLDVSHNRLVELPPASLATYPGLVHLDVGFNSLTKLEDSLCOTLGLLRTLTVOHNEVH LTSLDVSHNRLRGIPPESLRPYPGLLHLSVSYNTIAKLDGRLCETLPRLQTLDVAHNQVL ITVLNLTHNOLRRLPAANFTRYSQLTSLDVGFNTISKLEPELCOKLPMLKVLNLOHNELS $:^{*}:::^{* *}:^{*} \quad:^{*}, .:^{*},{ }^{*}{ }^{*},{ }^{*},:^{*}:::^{* *}:{ }^{* *}: .^{*}{ }^{*}: .^{*}:{ }^{* *}::$

GLTEEDLSNCTNLTELNLAGN-RLKLRGDPFAALQSLTLLDVSKNDLKTAKLGTRLQLPS WLKEKDLSNCTNLTELNLAGN-RLKLRGEPFAALOSLTLLDVSKNDLKTANLGTRLQLPS ALREEDLSRCSGLTAL ILRSN-RLKLQGEPFSGLQKLTYLDVSLNNLQSARLGSRPQLPA QLSDKTFAFCTNLTELHLMSNSIOKIKNNPFVKOKNLITLDLSHNGLSSTKLGTQVQLEN

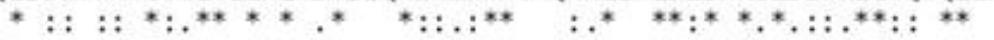

LVTLILSSNSISTIKKDDFSFLRNSSSLRVLHLSDLITLAKFEPDCLKPIASIYELVMNG LVTLILSSNSISTFKKDDFYFLSNSSSLRVLHLSNLTTLAKFEPDCLKPIASIYELVMNG LVNLSLAQNGITVLKRDDFSFLNHSSSLRVLNLSSVP - LKTLEPGCLKPISGLSTLILDG LQELLLSNNKIQALKSEELDIFAN-SSLKKLELSSNQ-IKEFSPGCFHAIGRLFGLFLNN

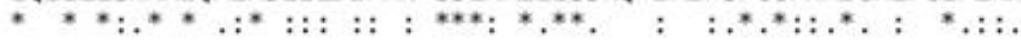

SKLGPSLTSKLCTQLSGTAIRSLSLQKTQLVTQDNTTFKGLGKTHLTTLDLSHNSIAKIG SKLGPSLTSKLCTELSGTAIRSLSLOKTQLVTLDNTTFKGLGKTHLTTLDLSHNSIAKIG SNMGALVIAGICSQLSGTAISSLSLRNMKLASLPNTTFAGLQQTNLSCLDLSRNGLGKIE VQLGPSLTEKLCLELANTSIRNLSLSNSQLSTTSNTTFLGLKWTNLTMLDLSYNNLNVVG $::^{*} .::^{*}:^{*}: .^{*}:^{*} . * * *::^{*}:{ }^{* * * * * * *} *^{* *}::^{* * * *} * .: \quad:$

DGSFOWLPMLEVLSLEQNNLKHLTKNTFHGLGNLTRLNLNMALVKS - RTSSYPIIDDFS DGSFOWLSMLEVLSLEONNLKRLTKNTFNGLGNLTRLNLNMALVKS - - RTSSYPIIDDFS NGSFOWLPRLETLILMENNIKHLTRDTFQGLQSLKTLQLTKALVKG - -HTSATPIIDNFS NDSFAWLPQLEYFFLEYNNIOHLFSHSLHGLFNVRYLNLKRSFTKOSISLASLPKIDDFS $: .^{* * * *},{ }^{* *}:{ }^{*}{ }^{* *}::^{*} \quad .::^{* *},:^{*}:^{*},::^{*} \quad::^{* * *}:^{* *}$

FQPLGALESLSMENTAFRNISVFTFAGLMSLRQLHLSGTSCMANVFSLRIITNQTFVSLA FQPLRALESLSMENTAFRNISVLTFAGLMSLRQLHLSRTSCMS - .. - LKIITNQTFVSLA FQPLTTLESLVLQKTAIREITTOTFTGLTSLKELDLSWSSCLS. - . - LKTITNETFLSLA FQWLKCLEHLNMEDNDIPGIKSNMFTGLINLKYLSLSNSFTS - ... - LRTLTNETFVSLA

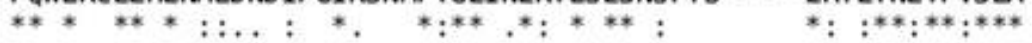

DSPLLTLKLTRTAISRLDPGAFSSLGNLTTFLLGNNSISQALTGKEFQGLGQLQEIYLSN GSPLLMLKLTSTAISRLDPGAFSSLGNLTTLLLGNNSISQTLTGKEFQGLGQLQEIYLSN ASPLRKLNLTGAAVVQISPGGFSTLKSLTVLLLDSNFIKQTLTGREFEGLGQLEEIHMSL HSPLHILNLTKNKISKIESDAFSWLGHLEVLDLGLNEIGQELTGQEWRGLENIFEIYLSY

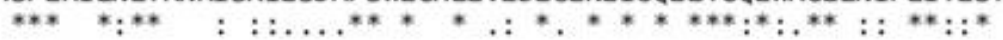

GNQKIILSPMSFVHVPALRTLMLGRALTGT-LYLNSSPFKPLSNLTILDLSNNNIANIKI GNOKLILSPMSFVHVPALRTLMLGRALTST-LYMNTSPFKPLSNLTILDLSNNNIANIKI NFOKVNLSSASFAAVPRLKVLTLGKSLTSTALNVDPSPFSPLVNLTFLDLSNNNIANIRR N-KYLQLTRNSFALVPSLQRLMLRRVALKN - VDSSPSPFQPLRNLTILDLSNNNIANIND

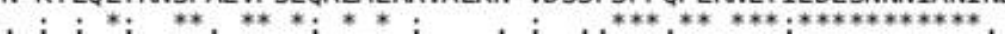

Figure 2. Alignment of ssTLR3 to corresponding trout (om), fugu (tr), and human (hs) sequences. Dashes indicate gaps inserted into the alignment.

Continued on next page

Genetics and Molecular Research 14 (2): 6073-6083 (2015)

CFUNPEC-RP www.funpecrp.com.br 
Figure 2. Continued.

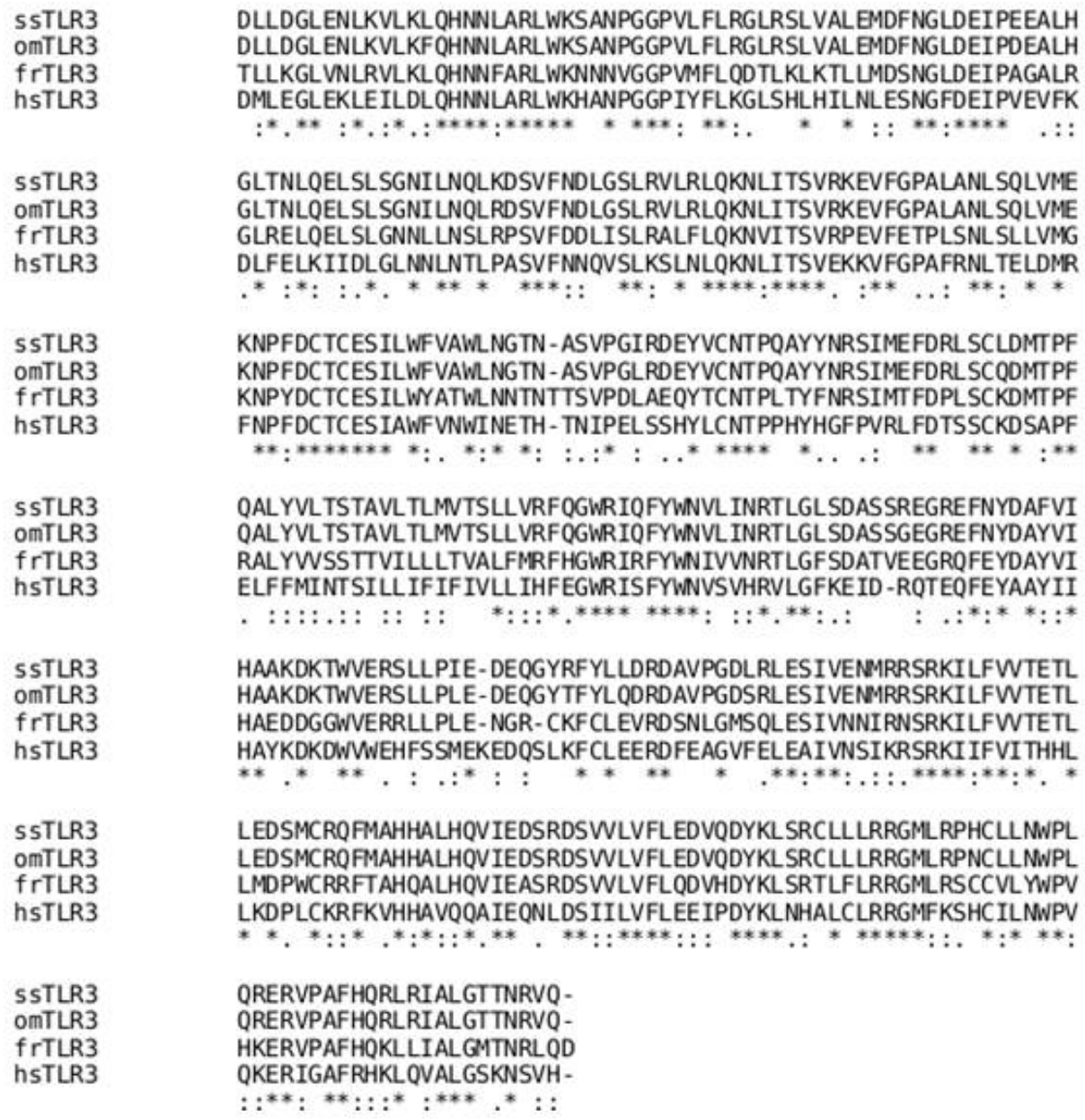

HAAKDKTWVERSLLPIE-DEQGYRFYLLDRDAVPGDLRLESIVENMRRSRKILFWTETL HAAKDKTWVERSLLPLE-DEQGYTFYLQDRDAVPGDSRLESIVENMRRSRKILFWTETL HAEDDGGWVERRLLPLE-NGR-CKFCLEVRDSNLGMSQLESIVNNIRNSRKILFWTETL HAYKDKDWWWEHFSSMEKEDQSLKFCLEERDFEAGVFELEAIVNSIKRSRKI IFVITHHL

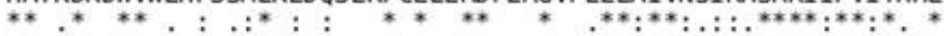

LEDSMCROFMAHHALHQVIEDSRDSWLVFLEDVQDYKLSRCLLLRRGMLRPHCLLNWL LEDSMCROFMAHHALHOVIEDSRDSWVVFLEDVQDYKLSRCLLLRRGMLRPNCLLNWPL LMDPWCRRFTAHQALHOVIEASRDSWVVFLQDVHDYKLSRTLFLRRGMLRSCCVL WPV LKDPLCKRFKVHHAVQQAIEONLDSIILVFLEE IPDYKLNHALCLRRGMFKSHCILNWPV

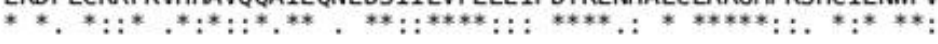

SSTLR3 QRERVPAFHQRLRIALGTTNRVQ-

OITLR3 QRERVPAFHQRLRIALGTTNRVQ

frTLR3 HKERVPAFHQKLLIALGMTNRLQD

hSTLR3 QKERIGAFRHKLQVALGSKNSVH-

$::^{* *}: * *:::^{*}::^{* * *} . *$ : :

\section{Amino acid alignment and phylogenetic analysis}

ssTLR3 showed important amino acid sequence similarity with trout (96.6\%), catfish (74.5\%), fugu (74.2\%), and zebrafish (74\%) TLR3 genes and low similarity with mammalian genes (Table 1). Phylogenetic analysis based on amino acid sequences clustered ssTLR3 within the same clade as other teleost and vertebrate TLR3 peptides (Figure 3). The high similarity of ssTLR3 to trout TLR3 (Skjaeveland et al., 2008) suggests that they are orthologs.

\section{Expression of ssTLR3 mRNA in different tissues}

ssTLR3 mRNAs were expressed in all organs and tissues examined with higher levels observed in the head kidney and spleen than in the other organs (Figure 4). 


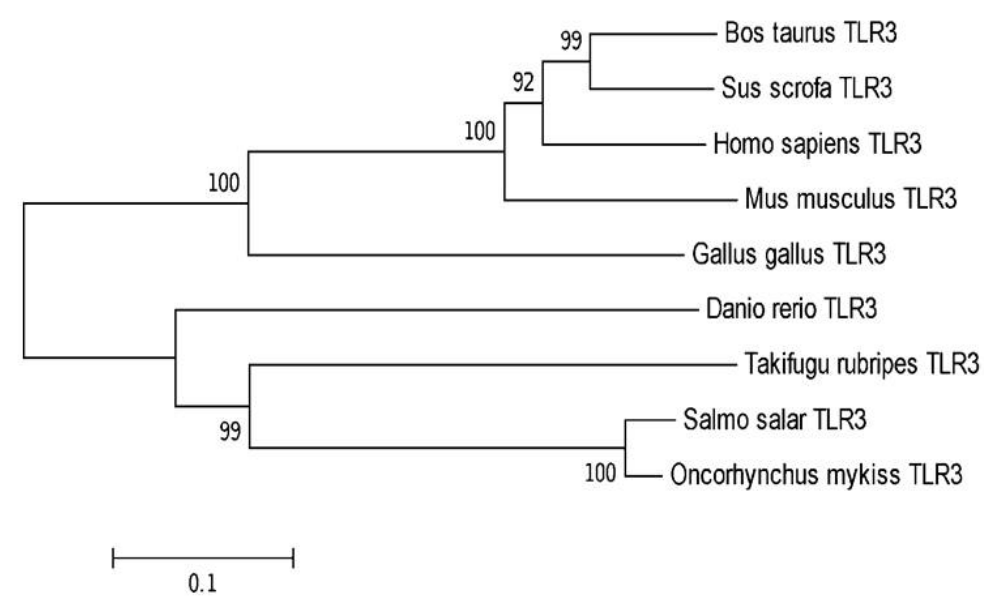

Figure 3. Phylogenetic tree of TLR3. Accession numbers of the sequences are given in Table 1. The gene tree was reconstructed using the neighbor-joining method. Values in the branch points correspond to bootstrap values.

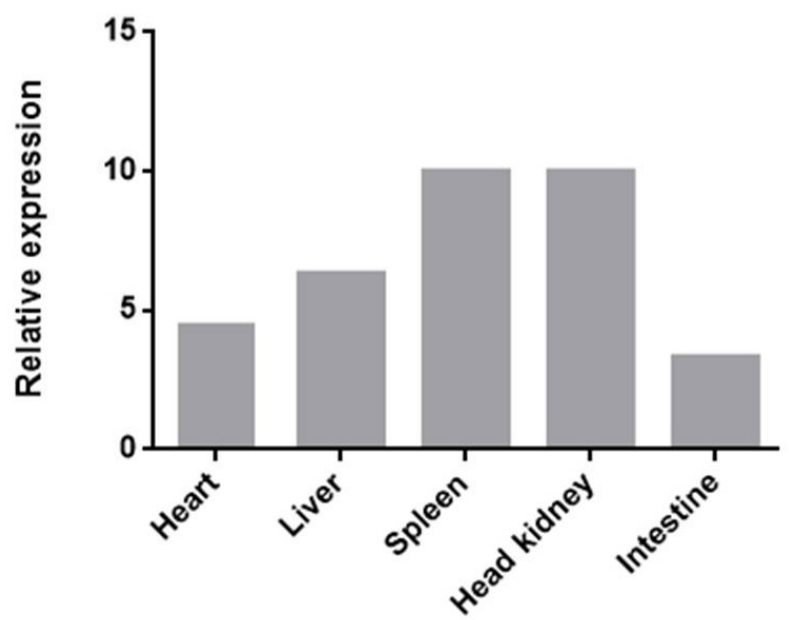

Figure 4. Gene expression analyses of the ssTLR3 gene in a range of organs from adult fish. Relative expression represents expression of pooled RNA samples from three Atlantic salmon. The results are reported as relative to expression ratios of the target gene in muscle; the tissue with the lowest expression.

\section{Expression of ssTLR3 in fish challenged with IPNV and Poly I:C stimulation}

The expression of ssTLR3 following Poly(I:C) stimulation was detected using realtime qPCR. After Poly(I:C) stimulation, ssTLR3 expression increased significantly in the head kidney of the fish (data not shown). The expression of ssTLR3 in the head kidney of individual fish inoculated with IPNV was also ascertained using real-time qPCR. As shown in Figure 5, the expression of ssTLR3 in the head kidney was significantly up-regulated between 5 and 21 days after IPNV inoculation. 


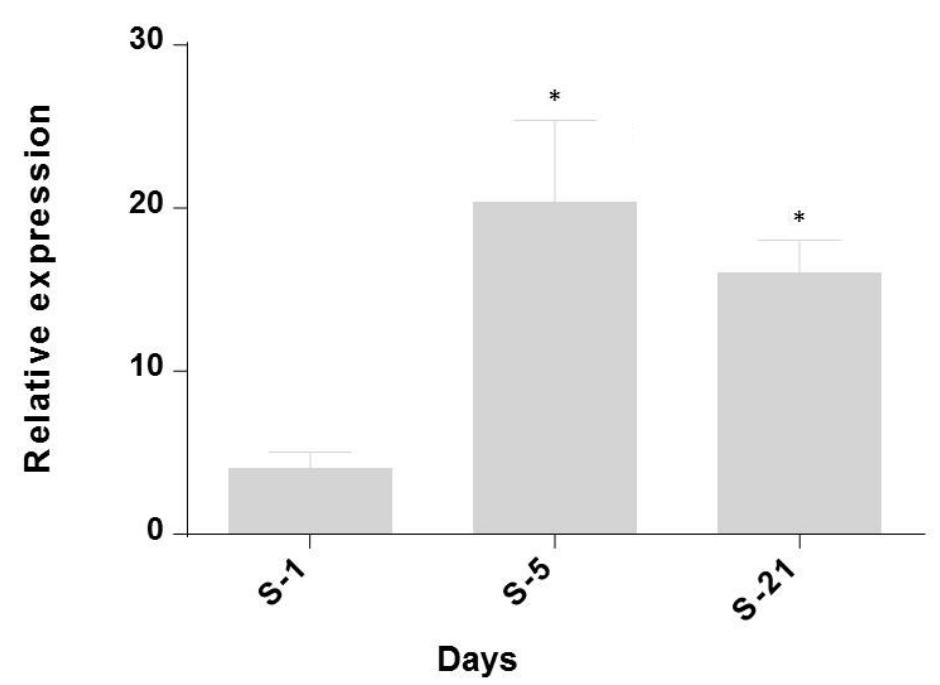

Figure 5. Quantitative analyses of distribution of the ssTLR3 gene in the head kidney of adult fish challenged with infectious pancreatic necrosis virus. The results are reported as the relative expression ratios to the control (unchallenged) \pm standard error. $* \mathrm{P}<0.05$.

\section{DISCUSSION}

The study presented here describes the identification and characterization of the putative ssTLR3 gene in the Atlantic salmon genome, its tissue expression distribution, and induced expression upon activation following Poly I:C stimulation and upon a single viral challenge.

The genomic ordering of the ssTLR3 coding region is very close to that of TLR3 genes from humans and fishes (e.g., zebrafish and rainbow trout), being organized in a similar manner across four exons. Similar to model vertebrates and rainbow trout TLR3s, the ssTLR3 proximal promoter region includes an ISRE, which is necessary for basal and IFN- $\beta$-induced promoter activation in murine and human TLR3s (Heinz et al., 2003). However, in contrast with the promoter regions described in rainbow trout and murine TLR3 genes (TATA-less) (Heinz et al., 2003; Rodriguez et al., 2005), we found a putative TATA box in the proximal promoter region 29 bp upstream of the transcription start point of ssTLR3. Recently, Zou et al. (2011) demonstrated a predominance of TATA box promoter gain after gene duplication events in genes associated with stress responses. The common ancestor of salmonids is purported to have experienced a whole genome duplication event between 25 and 100 mya (Ohno, 1970). However, at present, salmonids are assumed to be pseudo-tetraploid because they are in the process of reverting to a stable diploid condition. Therefore, we suggest that the presence of the TATA box in duplicate promoters may have a relevant function in ssTLR3 duplicate conservation by intensifying the expression distinction that may promote the adaptive evolution of salmonids in response to environmental variation.

The ssTLR3 receptor's ectodomain has 16 LRRs, which is similar to the number observed in zebrafish (Phelan et al., 2005) and human (Choe et al., 2005) TLR3s and the same as observed in rainbow trout (Rodriguez et al., 2005). The differences observed between human and zebrafish TLR3s may be associated with the different bioinformatic predictor software 
used. The TIR region represents amino acids known to have a relevant role in receptor signaling and in the intracellular localization (Sarkar et al., 2003; Funami et al., 2004) process. Funami et al. (2004) described three-amino acid residues relevant for human ligand activation, NF- $\kappa$ B, and IFN- $\beta$ promoter induction (Phe732, Leu742, and Gly743), which were observed in the ssTLR3 sequence. Sarkar et al. (2003) recognized particular tyrosine residues in the TIR region as being relevant for intracellular signaling, with the residue Tyr759 being the most decisive. This residue is present in ssTLR3 (Tyr766). Other tyrosine residues, such as Tyr733 or Tyr858, are required for maximal activity of the 561-gene promoter in humans, in which activation by dsRNA is mediated by TLR3. Both are present in ssTLR3 (Tyr742 and Tyr866, respectively). Supplementary tyrosine residues (Tyr756 and Tyr764) can also participate in the function of Tyr759, although they are less effective. Of these, only Tyr756 is conserved in Atlantic salmon (Tyr769). Funami et al. (2004) recognized two amino acids relevant for intracellular localization of the human TLR3, Arg740, and Val741. The arginine residue is conserved in Atlantic salmon (Arg750), rainbow trout, zebrafish, and fugu TLR3 sequences. However, the Val residue was not observed in the ssTLR3 sequence and the significance or implications of this missing residue are currently unclear.

TLR3 has been characterized in several fish species and mammals, and has been implicated in the identification of double-stranded RNA (Alexopoulou et al., 2001), which is made during replication of several viruses (Jacobs and Langland, 1996; Sen and Sarkar, 2005). The tissue expression profile of ssTLR3 was similar to that reported in zebrafish and rainbow trout TLR3 (Meijer et al., 2004; Rodriguez et al., 2005), showing expression in a wide range of tissues. This is an important result because differences in TLR3 expression patterns have been reported. For example, Oshiumi et al. (2003) demonstrated limited TLR3 expression in the liver and digestive organs of fugu. TLR3 stimulation provokes cytokine production, particularly type I IFNs (Alexopoulou et al., 2001; Hoebe et al., 2003), through the activation of NF-kB, MAP kinases, and IRF3. Therefore, TLR3 differs from all other TLRs in that it does not use the MyD88 subordinate signaling pathway. Although generally TLR3 has been seen as an important driving force of TLR-mediated Th1 response (Pulendran, 2004), there is evidence that TLR3 is not entirely essential for efficient antiviral immunity (Edelmann et al., 2004; Wang et al., 2004). Our results demonstrate an increase in expression of ssTLR3 following a challenge with the IPNV, which indicates that ssTLR3 may be involved in the early response against infection in Atlantic salmon, mainly through activation of interferon and inflammatory pathways.

\section{ACKNOWLEDGMENTS}

Research supported by the CORFO-INNOVA, Chile (grant \#12IDL2-16192).

\section{REFERENCES}

Akira S and Takeda K (2004). Toll-like receptor signaling. Nat. Rev. Immunol. 4: 499-511.

Alexopoulou L, Holt AC, Medzhitov R and Flavell RA (2001). Recognition of double-stranded RNA and activation of NF-kappaB by Toll-like receptor 3. Nature 413: 732-738.

Altschul SF, Gish W, Miller W, Myers WA, et al. (1990). Basic Local Alignment Search Tool. J. Mol. Biol. 215: 403-410.

Bell JK, Mullen GE, Leifer CA, Mazzoni A, et al. (2003). Leucine-rich repeats and pathogen recognition in Toll-like receptors. Trends Immunol. 24: 528-533.

Cao B, Porollo A, Adamczak R, Jarrel M, et al. (2006). Enhanced recognition of protein transmembrane domains with prediction-based structural profiles. Bioinformatics 22: 303-309.

Cepeda V, Cofre C, González R, MacKenzie S, et al. (2011). Identification of genes involved in immune response of Atlantic 
salmon (Salmo salar) to IPN virus infection, using expressed sequence tag (EST) analysis. Aquaculture 318: 54-60.

Choe J, Kelker MS and Wilson IA (2005). Crystal structure of human Toll-like receptor 3 (TLR3) ectodomain. Science 309: 581-585.

Cofre C, Gonzalez R, Moya J and Vidal R (2014). Phenotype gene expression differences between resistant and susceptible salmon families to IPNV. Fish Physiol. Biochem. 40: 887-896.

Edelmann KH, Richardson-Burns S, Alexopoulou L, Tyler KL, et al. (2004). Does Toll-like receptor 3 play a biological role in virus infections? Virology 322: 231-238.

Funami K, Matsumoto M, Oshiumi H, Akazawa T, et al. (2004). The cytoplasmic 'linker region' in Toll-like receptor 3 controls receptor localization and signaling. Int. Immunol. 16: 1143-1154.

Heinemeyer T, Wingender E, Reuter I, Hermjakob H, et al. (1998). Databases on transcriptional regulation: TRANSFAC, TRRD and COMPEL. Nucleic Acids Res. 26: 364-370.

Heinz S, Haehnel V, Karaghiosoff M, Schwarzfischer L, et al. (2003). Species-specific regulation of Toll-like receptor 3 genes in men and mice. J. Biol. Chem. 278: 21502-21509.

Hoebe K, Du X, Georgel P, Janssen E, et al. (2003). Identification of Lps2 as a key transducer of MyD88-independent TIR signaling. Nature 424: 743-748.

Jacobs BL and Langland JO (1996). When two strands are better than one: the mediators and modulators of the cellular responses to double-stranded RNA. Virology 219: 339-349.

Kawai T and Akira S (2007). TLR signaling. Semin. Immunol. 19: 24-32.

Letunic I, Doerks T and Bork P (2009). SMART 6: recent updates and new developments. Nucleic Acids Res. 37: D229-D232.

Magnadottir B (2006). Innate immunity of fish (overview). Fish Shellfish Immunol. 20: 137-151.

Matsushima N, Tanaka T, Enkhbayar P, Mikami T, et al. (2007). Comparative sequence analysis of leucine-rich repeats (LRRs) within vertebrate Toll-like receptors. BMC Genomics 8: 124-143.

Medzhitov R (2007). Recognition of microorganisms and activation of the immune response. Nature 449: 819-826.

Meijer AH, Gabby Krens SF, Medina Rodriguez IA, He S, et al. (2004). Expression analysis of the Toll-like receptor and TIR domain adaptor families of zebrafish. Mol. Immunol. 40: 773-783.

Ohno S (1970). Evolution by Gene Duplication. Springer-Verlag, New York.

Oshiumi H, Tsujita T, Shida K, Matsumoto M, et al. (2003). Prediction of the prototype of the human Toll-like receptor gene family from the pufferfish, Fugu rubripes, genome. Immunogenetics 54: 791-800.

Palti Y (2011). Toll-like receptors in bony fish: from genomics to function. Dev. Comp. Immunol. 35: 1263-1272.

Petersen TN, Brunak S, von Heijne G and Nielsen H (2011). SignalP 4.0: discriminating signal peptides from transmembrane regions. Nat. Methods 8: 785-786.

Pfaffl MW (2001). A new mathematical model for relative quantification in real-time RT-PCR. Nucleic Acids Res. 29: e45-e51.

Phelan PE, Mellon MT and Kim CH (2005). Functional characterization of full-length TLR3, IRAK-4, and TRAF6 in zebrafish (Danio rerio). Mol. Immunol. 42: 1057-1071.

Pulendran B (2004). Modulating vaccine responses with dendritic cells and Toll-like receptors. Immunol. Rev. 199: 227-250.

Rebl A, Siegl E, Koollner B, Fischer U, et al. (2007). Characterization of twin Toll-like receptors from rainbow trout (Oncorhynchus mykiss): evolutionary relationship and induced expression by Aeromonas salmonicida salmonicida. Dev. Comp. Immunol. 31: 499-510.

Rodriguez MF, Wiens GD, Purcell MK and Palti Y (2005). Characterization of Toll-like receptor 3 gene in rainbow trout (Oncorhynchus mykiss). Immunogenetics 57: 510-519.

Sarkar SN, Smith HL, Rowe TM and Sen GC (2003). Double-stranded RNA signaling by Toll-like receptor 3 requires specific tyrosine residues in its cytoplasmic domain. J. Biol. Chem. 278: 4393-4396.

Schultz J, Milpetz F, Bork P and Ponting CP (1998). SMART, a simple modular architecture research tool: identification of signaling domains. Proc. Natl. Acad. Sci. U. S. A. 95: 5857-5864.

Sen GC and Sarkar SN (2005). Transcriptional signaling by double-stranded RNA: role of TLR3. Cytokine Growth Factor Rev. 16: 1-14.

Skjaeveland I, Iliev DB, Zou J, Jørgensen T, et al. (2008). A TLR9 homolog that is up-regulated by IFN-gamma in Atlantic salmon (Salmo salar). Dev. Comp. Immunol. 32: 603-607.

Tamura K, Peterson D, Peterson N, Stecher G, et al. (2011). MEGA5: molecular evolutionary genetics analysis using maximum likelihood, evolutionary distance, and maximum parsimony methods. Mol. Biol. Evol. 28: 2731-2739.

Wang T, Town T, Alexopoulou L, Anderson JF, et al. (2004). Toll-like receptor 3 mediates West Nile virus entry into the brain causing lethal encephalitis. Nat. Med. 10: 1366-1373.

Zou Y, Huang W, Gu Z and Gu X (2011). Predominant gain of promoter TATA box after gene duplication associated with stress responses. Mol. Biol. Evol. 28: 2893-904. 